\title{
Brief Contribution to Geometrical Theory of Zeroes and Poles of Bifractional Filters
}

\author{
Jiri Petrzela \\ Department of Radio Electronics \\ Brno University of Technology \\ Brno, Czech Republic \\ petrzelj@feec.vutbr.cz
}

\begin{abstract}
This paper briefly contributes to synthesis of non-integer order frequency filters. Well known method of construction of desired frequency responses based on locations of zeroes and poles is generalized to fractionalorder (FO) domain. Several prototypes of FO transfer functions are geometrically interpreted using a complex plane. Each transfer function has a single real degree of freedom and corresponding unique frequency responses. Proposed approach is verified by example of very simple two-band FO audio equalizer.
\end{abstract}

Keywords-audio filter; equalizer; frequency response; fractional order; constant phase element

\section{INTRODUCTION}

Despite a non-integer order calculus belongs to the old mathematical ideas, FO analog circuits still attract considerable interest of design engineers. Reason for this can be found in the fundamental properties of FO network element, namely constant phase shift between response and driving force. In upcoming text, current and voltage will be considered as response and driving force quantity such that FO capacitor becomes created. Simultaneously, math order of FO capacitor will be considered as a real number between zero and three, non-integer nature of circuit is represented by symbol $\alpha \in(0,1)$. Higher order FO capacitors can be designed by utilizing impedance multipliers, general impedance converters, etc.

A significant number of books and journal papers focused on signal processing applications of FO circuit elements have been already published [1]. Theoretical background dedicated to construction of FO capacitors for specific frequency range can be found in paper [2]. Numerical method provided there turns to be universal and was utilized to derive high precision FO capacitors for audio applications, see [3] for details.

\section{PROTOTYPES OF TRANSFER FUNCTIONS}

It is well known that, if plotted in a complex plane, locations of zeroes and poles of conventional integerorder network can be directly used to draw frequency responses. Such analog circuit can be hypothetically decomposed into a cascade of bilinear and biquadratic filtering sections. For each singular zero and/or pole module frequency response (MFR) is a ratio between two distances: ending point of vector $j \omega$ and position of a zero and/or pole, respectively. Note that this only holds for the lumped circuits with minimal argument. Phase frequency response (PFR) is difference between

Research described in this paper was financially supported by Grant Agency of Czech Republic through project no. 19-22248S. two angles. Angles can be specified by three points: zero, location of zero/pole and ending point of vector $j \omega$. Biquadratic filters and higher-order circuits can be considered only as generalization of this concept: zeroes and poles can be complex conjugated numbers and its location can be in open left half-plane of complex plane. Upcoming subsections of this paper reformulate geometrical approach mentioned above for singular zeroes and poles with different FO.

\section{A. Transfer with Total Order Less than One}

This kind of the two-port contains following term in numerator and/or denominator of transfer function in Laplace transform

$$
K(s)=\frac{X_{\text {out }}(s)}{X_{\text {in }}(s)}=s^{\alpha}+\omega_{0}^{\alpha},
$$

where $\omega_{0}^{\alpha}$ represents a significant angular frequency, $X_{\text {in }}$ and $X_{\text {out }}$ is a network quantity. Complex frequency response can be expressed as

$$
\begin{aligned}
K(j \omega)= & \omega^{\alpha} \cdot \cos \left(\frac{\pi}{2} \alpha\right)+\omega_{0}^{\alpha}+ \\
& +\left[\omega^{\alpha} \cdot \sin \left(\frac{\pi}{2} \alpha\right)\right] j .
\end{aligned}
$$

Having this formula, PFR can be easily obtained as

$$
\varnothing(\omega)=\operatorname{atan} \frac{\omega^{\alpha} \cdot \sin \left(\frac{\pi}{2} \alpha\right)}{\omega_{0}^{\alpha}+\omega^{\alpha} \cdot \cos \left(\frac{\pi}{2} \alpha\right)} .
$$

Both MFR (blue abscissa) and PFR (red angle) can be derived using plane plot provided in Fig. 1. This figure shows 3D graphs that relate MFR (length of line M) and PFR with a location of FO zero. An asymptotical phase change begins with zero and ends at $90 \alpha^{\circ}$.

\section{B. Transfer with Total Order Between One and Two}

This kind of the transfer function can be expressed using Laplace transform as

$$
K(s)=s^{1+\alpha}+\omega_{0}^{1+\alpha},
$$

where number $\omega_{0}^{1+\alpha}$ stands for a significant frequency. Complex frequency response is given as

$$
\begin{array}{r}
K(j \omega)=\omega_{0}^{1+\alpha}-\omega^{1+\alpha} \cdot \sin \left(\frac{\pi}{2} \alpha\right)+ \\
+\left[\omega^{1+\alpha} \cdot \cos \left(\frac{\pi}{2} \alpha\right)\right] j .
\end{array}
$$


By handling with this complex equation PFR can be derived, namely

$$
\varnothing(\omega)=\operatorname{atan} \frac{\omega^{1+\alpha} \cdot \cos \left(\frac{\pi}{2} \alpha\right)}{\omega_{0}^{1+\alpha}-\omega^{1+\alpha} \cdot \sin \left(\frac{\pi}{2} \alpha\right)} .
$$

Note that a "speed of change" of magnitude frequency response is increased if compared to previous kind of transfer function but lower than upcoming cases. Also note band-reject nature of module frequency response. Position of local minima depends on chosen value $\omega_{0}$. Numerical results are shown via Fig. 2. Asymptotical phase shift starts at $0^{\circ}$ and ends with $90(1+\alpha)^{\circ}$.

\section{Transfer with Order Between Two and Three}

This case can be expressed using a simple transfer function of the form

$$
K(s)=s^{2+\propto}+\omega_{0}^{2+\alpha},
$$

where real-valued $\omega_{0}^{2+\alpha}$ represents significant angular frequency. By substitution $j \omega \rightarrow s$ following complex frequency response can be obtained

$$
\begin{array}{r}
K(j \omega)=\omega_{0}^{2+\alpha}-\omega^{2+\alpha} \cdot \cos \left(\frac{\pi}{2} \alpha\right)- \\
-\left[\omega^{2+\alpha} \cdot \sin \left(\frac{\pi}{2} \alpha\right)\right] j,
\end{array}
$$

and using basic theory of the complex numbers PFR can be derived as

$$
\emptyset(\omega)=\operatorname{atan} \frac{\omega^{2+\alpha} \cdot \sin \left(\frac{\pi}{2} \alpha\right)}{\omega^{2+\alpha} \cdot \cos \left(\frac{\pi}{2} \alpha\right)-\omega_{0}^{2+\alpha}} .
$$

Numerical results associated with this specific transfer function are given by means of Fig. 3. MFR exhibits band-reject shape that can be utilized in many practical applications such as audio equalizers discussed here. Phase change begins with $0^{\circ}$ and ends at $-90(1+\alpha)^{\circ}$.

\section{Transfer with Order Between Three and Four}

This case can be expressed using a simple transfer function of the form

$$
K(s)=s^{3+\alpha}+\omega_{0}^{3+\alpha},
$$

where $\omega_{0}^{3+\alpha}$ is a real number that represents significant angular frequency. Corresponding complex transfer function having separated real and imaginary part is

$$
\begin{array}{r}
K(j \omega)=\omega_{0}^{3+\alpha}+\omega^{3+\alpha} \cdot \sin \left(\frac{\pi}{2} \alpha\right)- \\
-\left[\omega^{3+\alpha} \cdot \cos \left(\frac{\pi}{2} \alpha\right)\right] j .
\end{array}
$$

Fundamental theory of the complex numbers provides PFR described by following equation

$$
\emptyset(\omega)=-\operatorname{atan} \frac{\omega^{3+\alpha} \cdot \cos \left(\frac{\pi}{2} \alpha\right)}{\omega_{0}^{3+\alpha}+\omega^{3+\alpha} \cdot \sin \left(\frac{\pi}{2} \alpha\right)},
$$

where $\omega_{0}^{3+\alpha}$ is significant frequency. Numerical results connected with term (11) are provided within Fig. 4. Phase change starts at $0^{\circ}$ and ends at $90(1-\alpha)^{\circ}$.
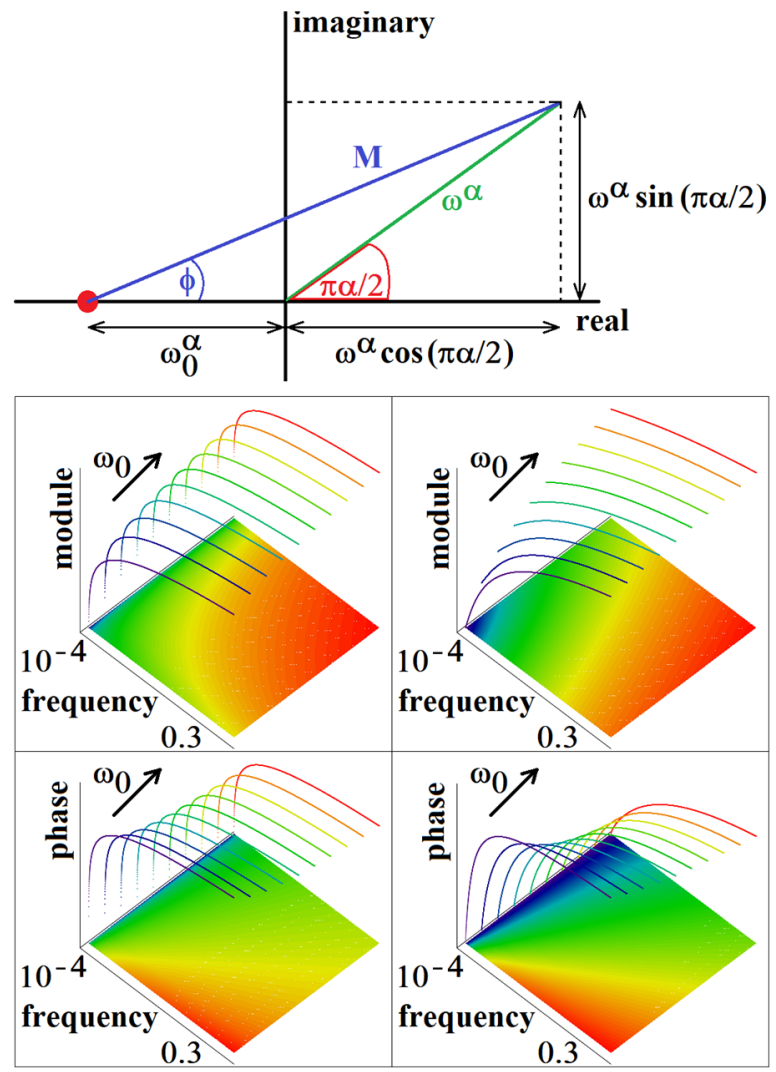

Figure 1. Fundamental transfer function with order between zero and one: principal geometry (upper plot), module (middle lots) and phase (lower graphs) frequency responses for $\omega_{0} \in(0.1,1)$ and math order $\alpha=0.2$ (left column) and $\alpha=0.8$ (right plots).
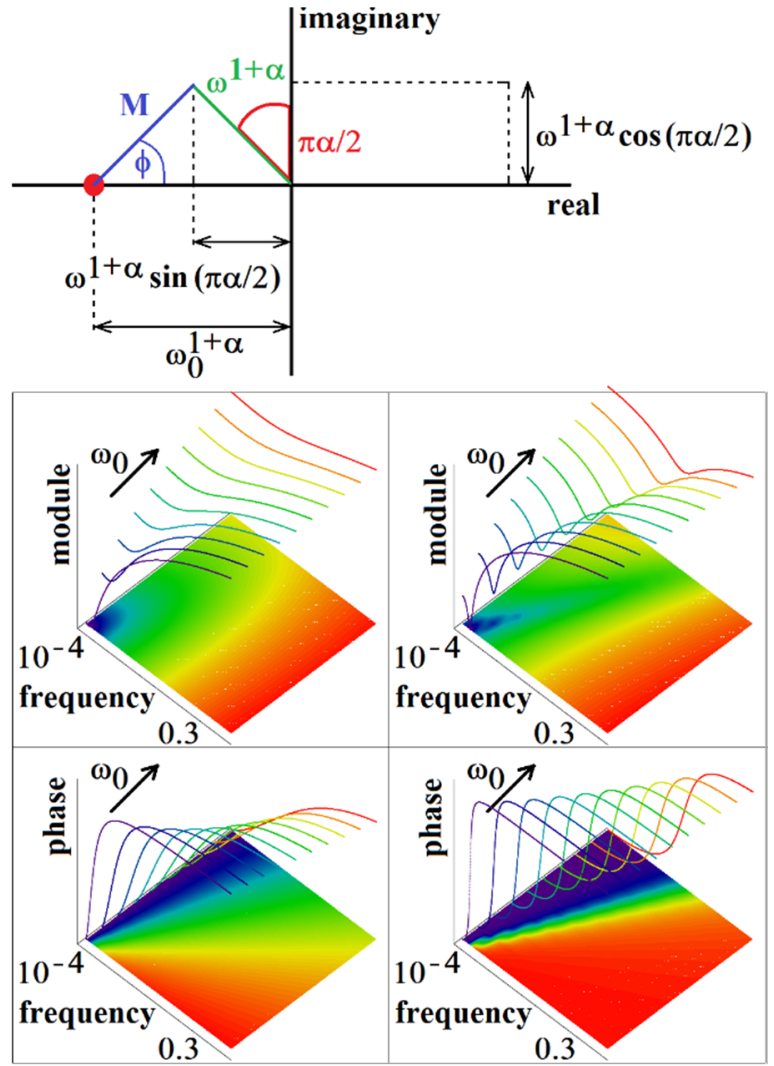

Figure 2. Order between one and two: geometry (upper plot), module (middle lots) and phase (lower graphs) frequency responses for $\omega_{0} \in(0.1,1)$ and FO $\alpha=0.2$ (left column) and $\alpha=0.8$ (right plots). 

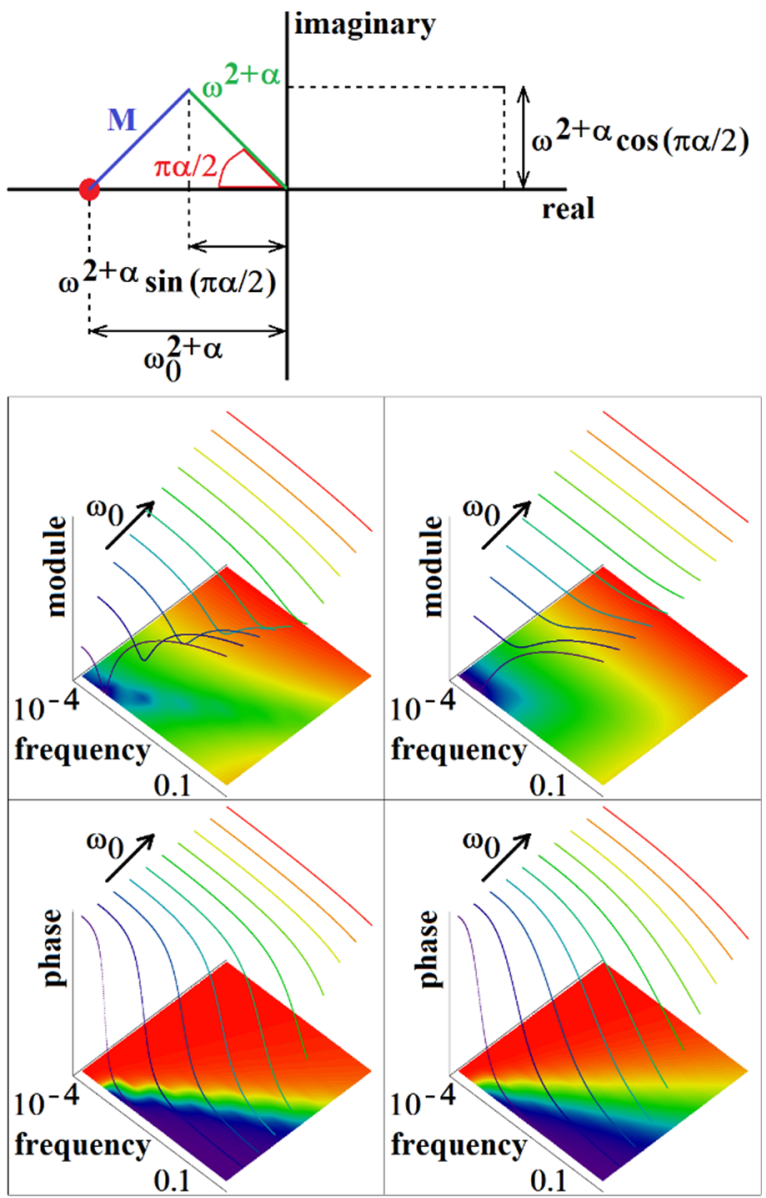

Figure 3. Fundamental transfer function with order between two and three: principal geometry (upper plot), module (middle lots) and phase (lower graphs) frequency responses for $\omega_{0} \in(0.1,1)$ and math order $\alpha=0.2$ (left column) and $\alpha=0.8$ (right plots).

\section{DESIGN OF SINGULAR FO ELEMENTS}

Probably the simplest approach to design higherorder FO circuit elements of form $1+\alpha$ is utilization of a general immittance converter (GIC). One example, application of well-known Antoniou's configuration, can be found in tutorial paper [4]. Procedure is simple and relies on existence of two-terminal approximations of FO capacitors in the frequency-domain [5].

1. Assume that individual branches of Antoniou's GIC are numbered in ascending order, starting with input, and ending with grounded impedance.

2. Two-terminal approximation of FO capacitor is connected as the last fifth impedance. If mathematical order between zero and one is desired all remaining impedances are resistors.

3. To construct FO admittance element of the form $1+\alpha$ conventional linear capacitor is connected as third impedance. All other impedances are resistors.

4. To construct FO admittance element of the form $2+\alpha$ conventional linear capacitor is connected as both first and third impedance. Remaining impedances are standard resistors.

5. To design $3+\alpha \mathrm{FO}$ circuit element first and third impedance will be capacitor, fourth will be inductor and second becomes resistor. Of course, inductor can be implemented by using additional GIC connected as synthetic inductance.
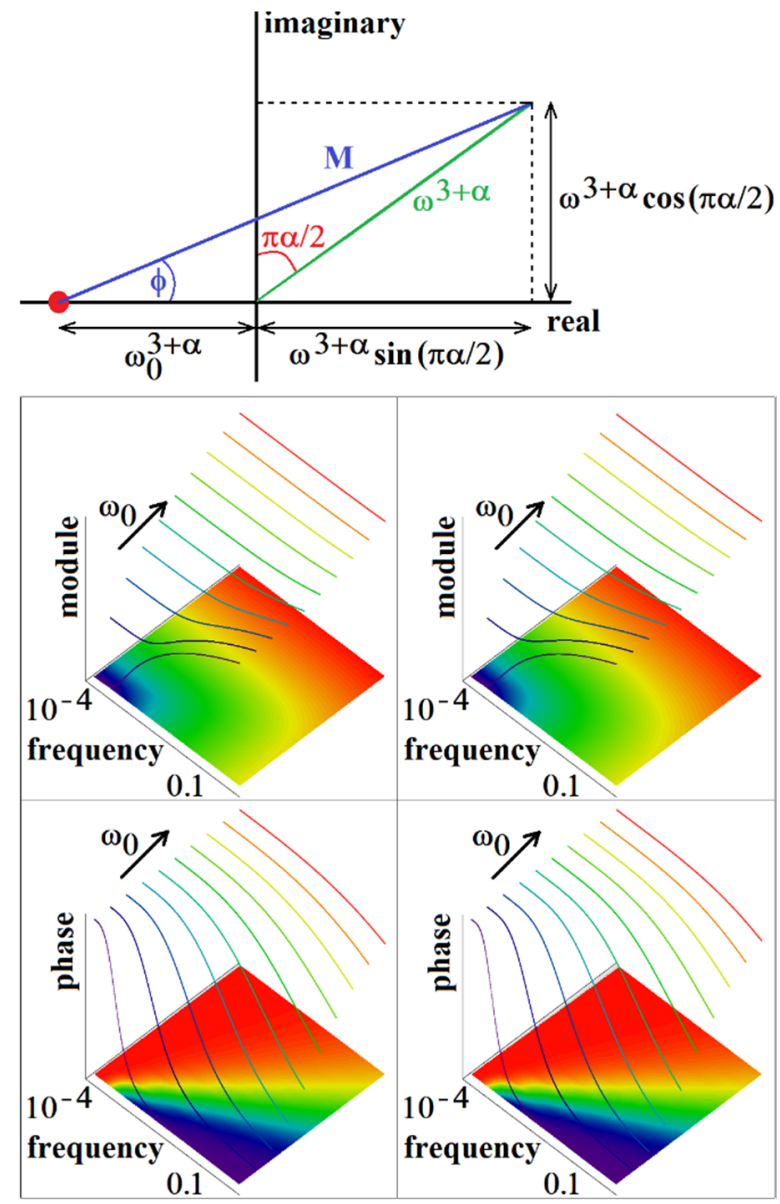

Figure 4. Fundamental transfer function with order between three and four: principal geometry (upper plot), module (middle lots) and phase (lower graphs) frequency responses for $\omega_{0} \in(0.1,1)$ and math order $\alpha=0.1$ (left column) and $\alpha=0.9$ (right plots).

Following this procedure, single GIC can model behavior up to $5+\alpha$ math order. Similarly, two GICs properly connected can model $4+\alpha$ without necessity to employ inductors. In the case of questions please do not hesitate to contact author for details. Drawback of GIC-like approach is evident: necessity to use supply voltage and many active elements.

\section{CONCEPT OF FO AUdIO EQUALIZER}

Idea behind multi-band FO audio equalizer is quite simple: use a band-reject nature of singular FO zeroes and poles of order $1+\alpha$ or $2+\alpha$. Synthesis of equalizer follows principle of a cascade connection of two-ports, namely first with FO transfer function

$$
K_{1}(s)=\frac{V_{\text {out }}}{V_{\text {in }}}=\frac{\omega_{0}^{2+\alpha}}{\omega_{0}^{1+\alpha}} \cdot \frac{s^{1+\alpha}+\omega_{0}^{1+\alpha}}{s^{2+\alpha}+\omega_{0}^{2+\alpha}}
$$

and second characterized by transfer function

$$
K_{2}(s)=\frac{V_{\text {out }}}{V_{\text {in }}}=\frac{\omega_{0}^{1+\alpha}}{\omega_{0}^{2+\alpha}} \cdot \frac{s^{2+\alpha}+\omega_{0}^{2+\alpha}}{s^{1+\alpha}+\omega_{0}^{1+\alpha}} .
$$

Numerical analysis of transfer (13) and (14), i.e. MFR, is demonstrated for specific parameters in Fig. 5. Note that both (13) and (14) resembles transfers of bilinear filters. However, a single bilinear filter is not able to amplify or attenuate specific frequency band. Circuitry realization of member (13) of group of the bifractional filters is provided in Fig. 6. This circuit was designed 
using matrix method of nodal voltages and is based on the operational transconductance amplifier (OTA) [6]. Suppose input and output node is numbered as one and three respectively and the remaining nodes marked as indicated in schematic. Then, voltage transfer function can be calculated as

$$
K(s)=\frac{V_{\text {out }}}{V_{\text {in }}}=\frac{\Delta_{1,3}}{\Delta_{1,1}}=-\frac{g_{m 2}}{C} \cdot \frac{s^{1+\alpha}+\frac{g_{m 1} \cdot g_{m 3}}{C \cdot Y_{\alpha}}}{s^{2+\alpha}+\frac{g_{m 1} \cdot g_{m 2} \cdot g_{m 4}}{C^{2} \cdot Y_{\alpha}}},
$$

where $Y_{\alpha}$ is the so-called pseudo capacitance and $\Delta_{k, n}$ is sub determinant of admittance matrix

$$
\mathbf{Y}=\left(\begin{array}{cccc}
Y_{\alpha} \cdot s^{\alpha} & -Y_{\alpha} \cdot s^{\alpha} & 0 & 0 \\
-Y_{\alpha} \cdot s^{\alpha} & Y_{\alpha} \cdot s^{\alpha} & 0 & g_{m 1} \\
0 & g_{m 2} & s \cdot C & 0 \\
g_{m 3} & 0 & g_{m 4} & s \cdot C
\end{array}\right)
$$

where default orientation of output current of OTAs is toward active elements.

Four differential input single output OTA elements are required for designed audio equalizer. Location of FO zero and pole can be adjusted independently via a trans-conductance $g_{m 3}$ and $g_{m 4}$ respectively. Constant $Y_{\alpha}$ depends on concrete approximation of FO capacitor and numerical values of the components used therein. Roughly speaking, it is equal to module of admittance measured at frequency $1 \mathrm{rad} / \mathrm{s}$, i.e. $159 \mathrm{mHz}$. Filtering two-port characterized by transfer function (14) can be derived using matrix method of nodal voltages as well.
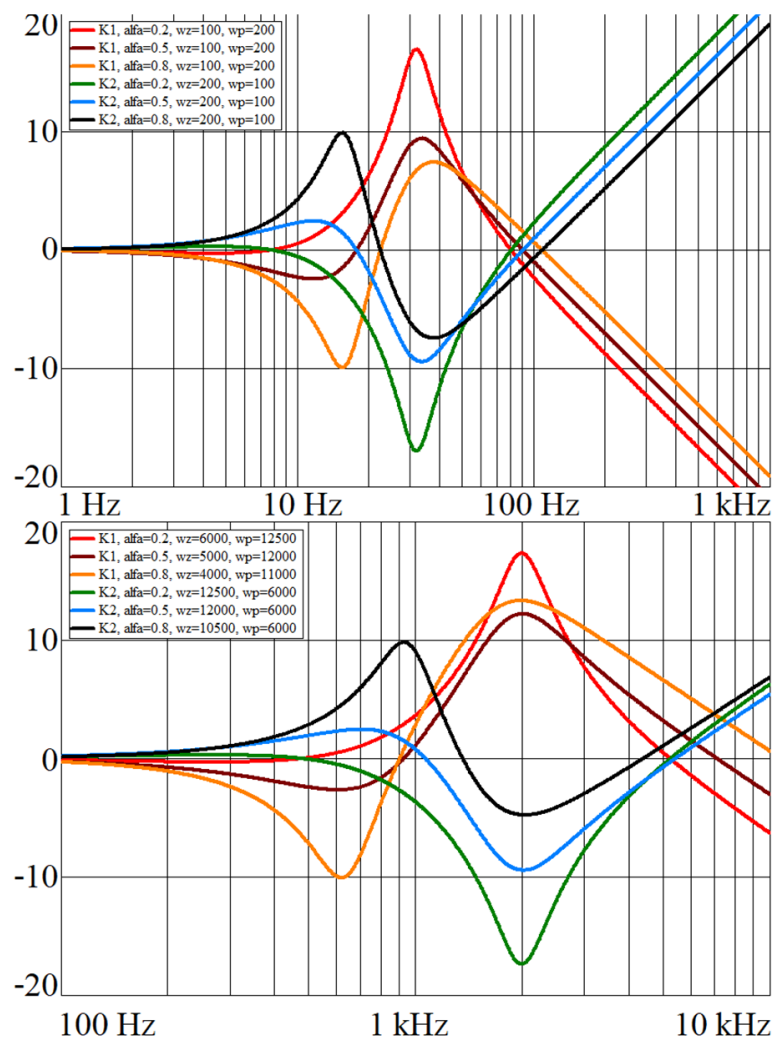

$00 \mathrm{~Hz}$

$1 \mathrm{kHz}$

$10 \mathrm{kHz}$

Figure 5. MFRs (dB vs frequency in $\mathrm{Hz}$ ) associated with a voltage transfer function (13) with numerical values specified as legends: bass band asymetric equalization with treble emphasis/attenutation (upper plots) and middle range equalization with consequent treble emphasis/attenuation (lower graph).

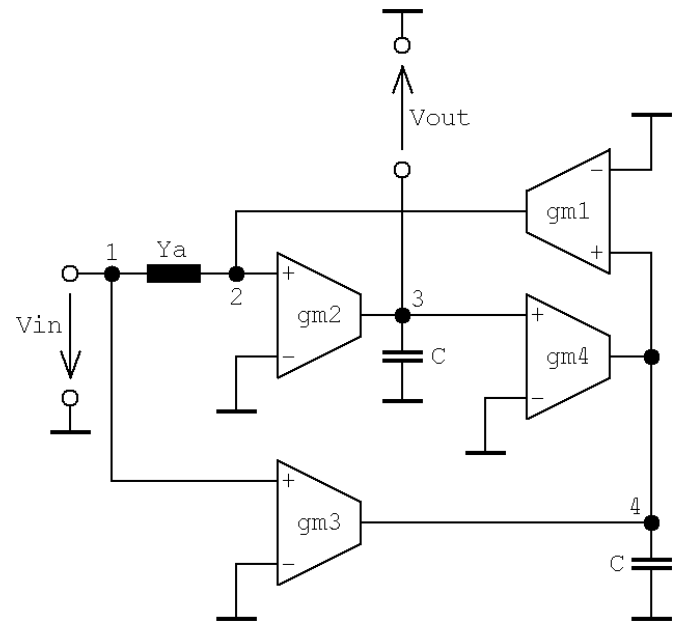

Figure 6. Circuit implementation of FO audio equalizer

\section{CONCLUSION}

This short study demonstrates the possibility to use geometrical interpretation of FO zeroes and poles to construct filtering section with the superior properties. One such example, namely acoustic equalizer, forms validation part of this paper. Individual parameters of equalizer are easily tunable via transconductances.

Despite lucidity, proposed graphical method does not solve troubles with specific frequency responses. Optimal transfer function of FO all-pass filter together with circuitry realization still waits to be discovered. Here we can find place for further research.

Note that polynomials having FO do not contain terms with all lower orders. For example, polynomial with respect to complex frequency $s$ having the highest order $2+\alpha$ does not contain order value $2,1+\alpha$ and $\alpha$. Considering a full-term polynomial, FO zero/pole can leave horizontal axis and move to open left half-plane of the complex plane. Detailed analysis of all possible situations is far beyond allowed extent of this paper.

Straightforward analysis proves that higher orders of a singular FO element such as $4+\alpha, 5+\alpha$, etc. need not to be solved. It is because repeated nature of power of the imaginary unit $j$, i.e. geometrical interpretations are the same as those provided in this paper.

\section{REFERENCES}

[1] M. D. Ortigueira, Fractional Calculus for Scientists and Engineers, $1^{\text {st }}$ ed., Springer, 2011.

[2] J. Valsa, P. Dovrak, and M. Friedl, "Network model of the CPE," Radioengineering, vol. 20, no. 3, pp. 619-626, 2011.

[3] J. Petrzela, "Accurate constant phase elements dedicated for analog signal processing," Applied Sciences, vol. 9, no. 22, pp. $4888,2019$.

[4] J. Petrzela, "Nonlinear resistor with Polynomial AV Characteristic and Its Application in Chaotic Oscillator," Radioengineering, vol. 13, no. 2, pp. 20-25, 2004.

[5] R. Sotner, L. Polak, J. Jerabek, and J. Petrzela, "Simple two operational transconductance amplifiers-based electronically controllable bilinear two port for fractional-order synthesis," Electronics Letters, vol. 54, no. 20, pp. 1164-1166, 2018.

[6] R. Sotner, J. Jerabek, N. Herencsar, R. Prokop, K. Vrba, and T. Dostal, "Resistor-less first-order filter design with electronically reconfiguration of it transfer function," In Proc. of $24^{\text {th }}$ Intern. Conference Radioelektronika 2014, 15-16 April 2014, Bratislava (Slovak Republic), pp. 63-66. 